\title{
Retrospective analysis of management of patients presenting with acute urinary retention due to benign prostatic hyperplasia: A hospital based study
}

\author{
Pandit RK ${ }^{1}$, Agrawal CS ${ }^{2}$, Chalise PR ${ }^{3}$, Sapkota $\mathbf{G}^{4}$
}

${ }^{1}$ Assistant Professor, ${ }^{2}$ Professor, ${ }^{4}$ Senior Resident, Department of Surgery, B.P. Koirala Institute of Health Sciences, Dharan, Nepal, ${ }^{3} \mathrm{MCh}$ Resident, Division of Urology, Tribhuban University Teaching Hospital, Kathmandu, Nepal

\begin{abstract}
Objectives: To analyze current practice of management of acute urinary retention (AUR) in men above 40 years of age at B.P.Koirala Institute of Health Sciences, Nepal.

Materials and methods: A total of 68 patients (aged 50-91 years) presenting with indwelling catheter for AUR were included in the study. Because of lack of clear guideline and limited health care facility in our setup trial without catheter (TWOC) was given selectively depending mainly on preexisting symptoms. AUR presumed to be due to urinary tract infection received only antibiotic and others diagnosed of benign prostatic hyperplasia (BPH) received alfa blocker $_{1}$ prior to TWOC. TWOC was given 3-15 days after such treatment. Those satisfied without catheter for at least a week were considered successful TWOC.

Results: The data was available for 68 patients (mean age 66.1 years). Of 15 patients treated with antibiotic alone, 11 $(73.3 \%)$ had successful TWOC. Of 57 with presumptive diagnosis of BPH, 68.9\% (31 of 45) had successful TWOC. Mean age, symptom score and prostate volume were higher for patients with unsuccessful TWOC.

Conclusion: Selective TWOC may be imperative to minimize unnecessary suffering from AUR in less easily accessible health care facilities like ours. It may also reduce total cost of treatment.
\end{abstract}

Key words: Acute urinary retention, benign prostatic hyperplasia, trial without catheter

\begin{abstract}
A UR is sudden and painful inability to pass urine ${ }^{1}$. It is a common problem and its incidence increases with age. Over 1 in 10 men in their 70s will experience acute urinary retention within the next five years ${ }^{2}$. BPH is the commonest cause of AUR. For relief, it requires immediate placement of indwelling transurethral or suprapubic catheter. Subsequent management includes either TWOC or early prostate surgery to get rid of catheter.
\end{abstract}

Studies have shown that urgent prostate surgery for AUR is associated with greater morbidity and mortality, in part owing to increased risk of sepsis and bleeding associated with catheterization ${ }^{3}$. Alternatively, successful TWOC may avoid surgery in some patients, or allow the surgery to be scheduled in the absence of a catheter ${ }^{4}$. Some patients may not agree for surgery. This increasingly led to TWOC in most of the centre.

Our general observation has been that, after AUR, many patients often pass through grievous sequence of events like inaccessibility to adequately trained health personnel in peripheries for catheterization, frequent iatrogenic urethral injuries during catheter placement and replacement, often complicating with haematuria and need for suprapubic catheterization, improper trials without catheter, need to stay with catheter for months and repeated change of catheter because of long waiting list of surgery, restricted freedom for work (as most patients are farmer and field workers) for livelihood and increased overall cost. Some patients would utter, "Death would be easier than life with this catheter".

The study was done with an aim to analyze management of patients with AUR by clinical judgment in our limited setup. We also explored possibility of avoiding alfa blocker in selected patents.

\section{Materials and methods}

The data were collected from records of patients involved in the prospective study "An observational study of patients with benign prostatic hyperplasia presenting at B. P. Koirala Institute of Health Sciences, Dharan,

\section{Correspondence}

Dr. Rakesh Kumar Pandit

Department of Surgery, BPKIHS, Dharan, Nepal

E-mail: rakeshkp1@yahoo.com 
Nepal" which was conducted from October 2004 through September 2005 (completed but unpublished). Seventy four men of 40 to 91 years had presented with spontaneous AUR. Precipitated AUR i.e., the one developing after some triggering events such as non-prostate related surgery, anaesthesia, medications with sympathomimetic or anticholinergic effects, or antihistamines were not included in the study because precipitated AUR is likely to have higher success rate of TWOC and less need for subsequent surgery ${ }^{5}$. AUR following neurogenic events like spine injury were also excluded.

The initial evaluation of patients included demographic profile, lower urinary tract symptoms (LUTS), International Prostate Symptom Score (IPSS) ${ }^{6}$, and other relevant medical (particularly neurologic and diabetes mellitus) and surgical history. Digital rectal examination was done to assess anal tone and approximate prostate size. Urine routine, microscopy and culture were done, and blood urea, serum creatinine, electrolytes and serum prostate specific antigen were measured. Transabdominal ultrasound was used to measure prostate volume and assess for evidences of bladder outlet obstruction (BOO) on the bladder and upper urinary tracts.

Intention was to treat. In the absence of clear guideline, whether to attempt TWOC or schedule for surgery, decision was made selectively depending mainly on duration and severity of pre-existing symptoms and patients' wish. Accordingly, those patients with higher symptom score were generally prepared for surgery electively. However attempt to void without catheter was made by some patients at the time of catheter replacement. Those with mild and moderate symptom score were generally given 1-2 attempts of TWOC. Patients with evidences of advanced BOO like azotemia and back-pressure effect on bladder and upper urinary tract did not have TWOC.

Most patients (with diagnosis of $\mathrm{BPH}$ ) received $3-15$ days of $\alpha_{1}$-blocker (terazocin, prazocin or tamsulocin) prior to TWOC. Some with no previous LUTS or very short history of predominantly irritative symptoms like burning micturition and frequency, and positive urine culture received only antibiotic prior to TWOC. For those with successful TWOC and improvement of symptoms, UTI was designated as the culprit for AUR retrospectively. So, the diagnosis of $\mathrm{BPH}$ was presumptive based on careful analysis of clinical features. TWOC was considered unsuccessful if recurrent AUR developed within a week of catheter removal. Those who had successful TWOC received further course of $\alpha_{1}$-blocker. Finesteride was also advised to those with larger prostate (generally $40 \mathrm{cc}$ or above).

The data was analyzed using SPSS $10 \mathrm{PC}^{+}$and presented in term of mean, range and standard deviation. For comparison of variables, ' $t$ ' test was applied to find out the level of significance. 'P' value of less than 0.05 was considered significant.

\section{Results}

Sixty eight patients had data available for analysis. The mean age was 66.1 (SD 9.4, range 50-91) years. Out of 15 patients treated with antibiotic alone, $11(73.3 \%)$ had successful TWOC, and were designated as having UTI. Among the remaining four patients, two were excluded and the other two had recurrent retention within a week. The latter two were presumed to have BPH. Of the 57 patients diagnosed of BPH, 45 had TWOC and 12 were scheduled for earliest possible elective surgery. Thirty one $(68.9 \%)$ patients had successful TWOC. Overall success rate was $70 \%$. Thirty five $(51.5 \%)$ of patients had positive urine culture. Among the BPH-patients who were subjected to TWOC, fourteen (45.1\%) and 8 (57 $\%$ ) of patients with successful and unsuccessful TWOC had positive urine culture at presentation respectively.

Among patients diagnosed of $\mathrm{BPH}$, mean age (SD), symptom score (SD) and prostate volume (SD) of patients having TWOC and not having TWOC were $66.8(9.27)$ and $66.7(10.4)$ years, $15.9(5.13)$ and 24.8 (3.56), and 53.1 (33.4) and 55.95 (15) cc respectively. Only symptom score had statistically significant difference in the two groups $(\mathrm{p}=.000)$ (Table 1).

Mean age (SD), symptom score (SD) and prostate volume (SD) of BPH-patients with successful and unsuccessful TWOC were 65.3 (9.5) and 70 (8.5) years, 14.9 (4.7) and 18.21 (5.7), and 47.4 (32.8) and 65.8 (33.7) cc respectively. Though means of all the three variables are higher for unsuccessful group, only difference in symptom score was statistically significant $(\mathrm{p}=.04)($ Table 2$)$.

Table 1: Variables in BPH-patients with and without TWOC

\begin{tabular}{|l|c|c|c|}
\hline & TWOC $(\mathbf{n}=\mathbf{4 5})$ & No TWOC $(\mathbf{n}=\mathbf{1 2})$ & P value \\
\hline Age (years; Mean (SD, range)) & $66.8(9.27,51-91)$ & $66.75(10.36,50-80)$ & .987 \\
\hline Symptom score (Mean (SD, range) & $15.9(5.13,4-28)$ & $24.8(3.56,19-30)$ & .000 \\
\hline Prostate volume (cc; Mean (SD, range)) & $53.1(33.4,20-159)$ & $55.95(14.99,34.5-85)$ & .779 \\
\hline
\end{tabular}


Table 2: Variables in BPH-patients with successful and unsuccessful TWOC

\begin{tabular}{|l|c|c|c|}
\hline & Successful $(\mathbf{n}=\mathbf{3 1})$ & Unsuccessful $(\mathbf{n}=\mathbf{1 4})$ & P value \\
\hline Age (years; Mean (SD, range)) & $65.3(9.5,51-82)$ & $70(8.5,58-91)$ & .117 \\
\hline Symptom score (Mean (SD, range) & $14.9(4.7,5-28)$ & $18.21(5.7,4$ to 28$)$ & .044 \\
\hline Prostate volume (cc; Mean (SD, range)) & $47.4(32.8,20-159)$ & $65.8(33.7,20-132)$ & .09 \\
\hline
\end{tabular}

\section{Discussion}

After relief of AUR with catheter, prime concern of patients is to get rid of the catheter at the earliest. Whether do emergency surgery or follow TWOC, either has limitation. Emergency surgery seems to be associated with greater morbidity and mortality. Pickard et al $^{3}$, in a large observational cohort study found that, compared with men having prostatectomy to relieve lower urinary tract symptoms, men who had emergency prostatectomy after acute urinary retention were at increased risk of intraoperative complications (relative risk 1.8, 95\% confidence interval) like need for transfusion, postoperative complications, hospital death. Additionally, it is difficult to adjust in our limited operation-theatre availability. TWOC, if not given properly, the sequence of events is often painful and expensive because most of the patients in our country do not have easy accessibility to appropriate health care set up and we don't have well established health insurance system. This makes us imperative to exercise TWOC selectively.

No consensus has so far been reached at predicting the patient at risk of AUR accurately. Multiple risk factors are implicated in development of AUR like LUTS, enlarged prostate and older age ${ }^{2}$. Djavan et al. ${ }^{7}$ showed that age greater than 75 years and drained volume of urine greater than one litre are more likely to develop recurrent retention after TWOC. Klarskov et al. ${ }^{8}$ found after TWOC recurrence is $90 \%$ for men with an initial peak urinary flow rate less than $5 \mathrm{ml} / \mathrm{s}$. We, without urodynamic study or even uroflowmetry, had to depend on clinical judgment. Pre-existing symptoms are probably most convincing for patients to formulate treatment plan. This was the reason for significant difference in symptom score of our patients with and without TWOC. Less consideration was given to age and prostate volume for decision making. However, those with unsuccessful TWOC tended to have higher age, symptom score and prostate volume compared to those with successful ones. It was concordance to Jacobsen et al's ${ }^{2}$ observation.

Very few patients had records of drained urine volume at catheterization because most of the patients were either referred from outside with indwelling catheter or it was placed in emergency by emergency doctor.
Previously AUR developing after previous lower urinary tract symptoms was considered to be an absolute indication for prostatectomy ${ }^{9}$. However, recent guidelines have stated that a first episode of AUR with a successful TWOC may not be a mandatory indication for BPH surgery ${ }^{10}$.

Success rate of TWOC is variable in different studies. Recurrent retention of $56 \%$ to $70 \%$ have been reported within the first week ${ }^{8}$. Hastie et al. ${ }^{11}$ estimated that up to $93 \%$ of patients will require surgery in the long term. These data have recently been challenged by the results of several observational studies showing that only $32 \%$ of patients with successful TWOC or even less will require prostatectomy within 8 to 24 months of followup, reinforcing the need to offer a TWOC in patients with a first episode of AUR ${ }^{12}$. Djavan et al. ${ }^{13}$ found that increasing the period of bladder drainage improved the chances of voiding when the catheter was removed. Successful voiding was achieved by $44 \%$ of men randomised to immediate removal, $51 \%$ randomised to removal after two days, and $62 \%$ randomised to removal after seven days. In other studies success rates of $23 \%$ to $30 \%$ have been reported for TWOC performed one to three days after an episode of AUR ${ }^{11,14,15}$.

Our $70 \%$ of success rate was achieved after catheter removal in $3-15$ days. Status of any persistent UTI after treatment of initial positive urine culture was unknown in most of our patients as the culture was not repeated unless clinical features of UTI persisted or recurred. As it is difficult to maintain follow up in our set up, long term outcome could not be measured. However, some patients who followed up at variable periods were mostly satisfied. Only three patients came to our attention in the next six months - one with recurrent urinary retention, another with recurrent UTI and the other with unsatisfactory relief of symptoms with medical treatment. These patients and others with unsuccessful TWOC were scheduled for surgery.

Preoperative catheterization for certain period has advantage ofmore successfulvoiding after prostatectomy and less likely to require a second procedure because of bleeding ${ }^{3}$. Longer drainage was particularly important for men who had retained volumes of urine greater 
than $1.31^{13}$. We had advised longer duration (at least 2 weeks) of catheter for those with evidence of advanced BOO.

Several studies have shown that alpha $a_{1}$-blockers improve the rate of successful TWOC 10,15-17. Alpha-blockers are used routinely before catheter removal and are even considered an appropriate treatment option in the American Urological Association guidelines ${ }^{10}$. McNeill et al. ${ }^{4,12}$ showed that after successful TWOC, continued use of alfuzocin beyond the acute phase reduced the need for BPH surgery during a six-month treatment period. PLESS study ${ }^{18}$ has shown that long term use of finesteride reduces the risk of surgery and acute urinary

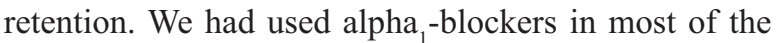
patents. In successful TWOC, we had continued alfa - $^{-}$ blocker for at least three months. Finesteride was added for bigger prostates (generally above $40 \mathrm{cc}$ ) for at least six months.

Some patients presumed to have only UTI were given only antibiotic, yet they had $73.3 \%$ successful TWOC. The reason for giving only antibiotic is that all AUR in patients of fifties and above may not necessarily be blamed to BPH, particularly when they did not have preexisting symptoms or have very short history of predominantly irritative symptoms like frequency and burning sensation. This information might have implication on the cost as long term use of medical treatment with alpha 1 -blockers and/or finesteride can be expensive for many poor patients. Seven patients had followed up a few days to weeks after catheter removal, and were all almost free of LUTS without medical treatment.

\section{Conclusion}

In conclusion, though routine TWOC is practiced in many centre, selective TWOC may be imperative to minimize unnecessary suffering in some patients in less easily accessible health care facilities like ours. It may also avoid unwanted cost of alfa-blocker and finesteride in some. Further prospective study should be conducted to explore potential advantage of selective TWOC.

\section{Acknowledgements}

To Dharnidhar Baral for stastical analysis.

\section{Refrence}

1. Emberton M, Anson K. Acute urinary retention in men: an age old problem. BMJ 1999; 318: 921-5.

2. Jacobsen SJ, Jacobsen DJ, Girman CJ, Roberts RO, Rhodes T, Guess HA, et al. Natural history of prostatism: risk factors for acute urinary retention. J Urol 1997; 158: 481-7.
3. Pickard R, Emberton M, Neal DE. The management of men with acute urinary retention. Br J Urol 1998; 81: 712-20.

4. McNeill SA, Hargreave TB, Roehrborn CG. Alfuzosin $10 \mathrm{mg}$ once a day in the management of acute urinary retention: results of a double blind placebo-controlled study. Urology 2005; 65: 83-90.

5. Roehrborn CG, Bruskewitz R, Nickel GC, et al. Urinary retention in patients with $\mathrm{BPH}$ treated with finasteride or placebo over 4 years. Characterization of patients and ultimate outcomes. The PLESS Study Group. Eur Urol. 2000; 37: 528-36.

6. Cockett ATK, AsoY, Chatelain C, Denis L, Griffiths K, Khoury S, et al. Recommendations of the international consensus committee. In: Proceedings of the international consultation on benign prostatic hyperplasia (BPH), 1991 Jun 26-7; Paris. P. 279-88.

7. Djavan B, Madersbacher S, Klingler C, Marberger M. Urodynamic assessment of patients with acute urinary retention: is treatment failure after prostatectomy predictable. J Urol 1997; 158: 1829-33 .

8. Klarskov P, Andersen JT, Asmussen CF, Brenoe J, Jensen SK, Jensen IL, et al. Symptoms and signs predictive of the voiding patterns after acute urinary retention in men. Scand J Urol Nephrol 1987; 21: 23-8.

9. McConnell JD - Benign prostatic hyperplasia: diagnosis and treatment. In: Rockville (ed) Clinical practice guideline number 8: Agency for health care policy and research, department of health and human services. US. AHCPR publication 94-0582; 1994.

10. AUA practice guidelines committee. AUA guideline on management of benign prostatic hyperplasia): diagnosis and treatment recommendation. J Urol 2003; 170: 530-47.

11. Hastie KJ, Dickinson AJ, Ahmad R, et al. Acute retention of urine: is trial without catheter justified? J R Coll Surg Edinb 1990; 35: 225-7.

12. McNeill SA, Hargreave TB, Gallagher H, et al. Long-term follow-up following presentation with a first episode of acute urinary retention (abstract). J Urol 2000;163: 307.

13. Djavan B, Shariat S, Omar M, Roehrborn CG, Marberger M. Does prolonged catheter drainage improve the chance of recovering voluntary voiding after acute retention of urine (AUR)? Eur Urol 1998; 33(suppl 1): 110.

14. Taube M, Gajraj H. Trial without catheter following acute retention of urine. BJU Int $1989 ; 63: 180-2$. 
15. McNeill SA, Daruwala PD, Mitchell ID, et al. Sustained-release alfuzosin and trial without catheter after acute urinary retention a prospective, placebo-controlled. BJU Int 1999; 84: 622-7.

16. McNeill SA, Donat R, Pillai MK, Hargreave TB, Daruwalla P, Goodman CM, et al. Prospective multicentre randomised placebo double blind study of the effect of alfuzosin on the outcome of trial removal of catheter following acute urinary retention. J Urol 1998;159: 256.
17. Lucas M, Stephenson TP, Nargund V. Tamsulosin reduces the need for recatheterisation following an episode of acute urinary retention in elderly benign prostatic hyperplasia patients (abstract). J Urol 2002; 167 (suppl): 266.

18. McConnell JD, Bruskewitz R, Walsh P, et al. The effect of finasteride on the risk of acute urinary retention and the need for surgical treatment among men with benign prostatic hyperplasia. Finasteride Long-Term Efficacy and Safety Study Group (comments). N Engl J Med 1998; 338: 557-63. 